\title{
An Efficient Method for Ground Maneuvering Target Refocusing and Motion Parameter Estimation Based on DPT-KT-MFP
}

\author{
Mingming Tian ${ }^{1, *}$, Guisheng Liao ${ }^{1}$, Shengqi Zhu ${ }^{1}$, Xiongpeng $\mathrm{He}^{1}$, Yongjun Liu ${ }^{1}$ and Yunpeng $\mathrm{Li}^{2}$ \\ 1 National Lab of Radar Signal Processing, Xidian University, Xi'an 710071, China; liaogs@xidian.edu.cn (G.L.); \\ sqzhu@xidian.edu.cn (S.Z.); xphe@xidian.edu.cn (X.H.); yjliu@xidian.edu.cn (Y.L.) \\ 2 Key Laboratory of Intelligent Perception and Image Understanding of Ministry of Education, \\ Xidian University, Xi'an 710071, China; yunpengli@stu.xidian.edu.cn \\ * Correspondence: mmingtian@stu.xidian.edu.cn; Tel.: +86-135-7290-2732
}

Citation: Tian, M.; Liao, G.; Zhu, S.; He, X.; Liu, Y.; Li, Y. An Efficient Method for Ground Maneuvering Target Refocusing and Motion Parameter Estimation Based on DPT-KT-MFP. Remote Sens. 2021, 13, 1092. https://doi.org/10.3390/ rs13061092

Academic Editor: Piotr Samczynski

Received: 20 January 2021

Accepted: 9 March 2021

Published: 12 March 2021

Publisher's Note: MDPI stays neutral with regard to jurisdictional claims in published maps and institutional affiliations.

Copyright: (c) 2021 by the authors. Licensee MDPI, Basel, Switzerland. This article is an open access article distributed under the terms and conditions of the Creative Commons Attribution (CC BY) license (https:// creativecommons.org/licenses/by/ $4.0 /)$.

\begin{abstract}
The image of ground maneuvering targets may be defocused due to the Doppler ambiguity, high-order range migration (RM), and Doppler frequency migration (DFM) caused by the target's complex motions in a synthetic aperture radar (SAR) system. To settle these problems, an efficient algorithm based on discrete polynomial-phase transform (DPT), keystone transform (KT), and matched filtering processing (MFP) is presented for ground maneuvering target refocusing and motion parameter estimation in this paper. Firstly, the DPT is applied to transform the cubic phase into the quadratic phase and simultaneously eliminate the quadratic RM, cubic RM, and quadratic DFM. Furthermore, the Doppler ambiguity containing Doppler center blur and Doppler spectrum ambiguity is also dealt with effectively by introducing a very small fixed lay time after DPT operation. Then, the KT is performed to correct the linear RM. After that, the matched filtering function related to the target's equivalent third-order coefficient is constructed to compensate for the residual linear DFM in the range-time and slow-time domain. Lastly, a well-refocused image of the maneuvering target can be acquired, and the target's motion parameters can be estimated effectively. The proposed algorithm has high computational efficiency and possesses favorable refocusing performance and motion parameter estimation precision. Simulation and real data processing results prove the effectiveness of the presented algorithm.
\end{abstract}

Keywords: synthetic aperture radar; Doppler center blur; Doppler spectrum ambiguity; ground maneuvering target refocusing; motion parameter estimation

\section{Introduction}

Synthetic aperture radar (SAR), which can work on all time scales and in all weather conditions, is widely applied in the civil and military fields [1-9]. In practical applications, in order to improve the focusing performance and acquire a high-resolution SAR image, the synthetic aperture time is usually long. However, within the long integration time, the ground maneuvering target may be severely defocused because of the high-order range migration (RM) and Doppler frequency migration (DFM) induced by the target's complex motions. As a consequence, in order to obtain good maneuvering target imaging performance, the effects of RM and DFM should be first eliminated.

The maneuvering target's cross-track velocity may cause linear RM. The keystone transform (KT) [10-14] was presented to correct the linear RM by rescaling the azimuth slow-time axis. The axis rotation moving target detection (AR-MTD) [15] was developed to eliminate the linear RM by axis rotation. In [16-18], Xu et al. proposed the radon Fourier transform (RFT), which jointly searches the target's range and velocity to eliminate the linear RM. Furthermore, the frequency-domain deramp-keystone transform (FDDKT) [19] was presented to refocus the maneuvering target without any parameter searching procedures. Nevertheless, for a ground maneuvering target with along-track velocity and 
cross-track acceleration, the abovementioned methods are invalid since they cannot eliminate the quadratic RM and linear DFM caused by the along-track velocity and cross-track acceleration of maneuvering targets.

In order to deal with the quadratic RM and linear DFM, several valid algorithms were recently presented. The second-order KT (SOKT) [20-22] was proposed to remove quadratic RM via time-scale transform. The radon fractional Fourier transform (RFRFT) [23] was developed to correct the linear and quadratic RM by searching the velocity and acceleration of the maneuvering target and then integrating the target energy via FRFT. The secondorder Wigner-Ville distribution (SOWVD) method [24] was proposed to correct the RM via KT and a compensation function related to SAR platform velocity, before focusing the maneuvering target via SOWVD. Tian et al. proposed an algorithm based on a symmetric autocorrelation function and window Lv transform (SAF-WLVT) [25] to simultaneously remove the linear and quadratic RM via SAF operation, before effectively integrating the target energy via WLVT. In addition, the symmetric autocorrelation function and scaled Fourier transform (SAF-SFT) method [26] and the deramp SOKT phase difference (deramp-SOKT-PD) method [27] were presented to eliminate the quadratic RM and linear DFM without any motion parameter searching procedures. However, when a ground target maneuvers with an along-track acceleration, cubic RM and quadratic DFM may occur in a high-resolution SAR system. Consequently, the focusing performance of the aforementioned algorithms may deteriorate dramatically because they ignore the effects of cubic RM and quadratic DFM. In addition, the focusing performance may further deteriorate due to the Doppler ambiguity containing the Doppler center blur and Doppler spectrum ambiguity caused by the target's fast maneuvering motions.

To address the abovementioned problems, the generalized radon Fourier transform (GRFT) [28] was proposed to eliminate the high-order RM and DFM, as well as deal with the Doppler ambiguity. Although this method has optimal coherent accumulation performance and parameter estimation precision, the computational complexity of GRFT on the basis of four-dimensional (4D) parameter search is so huge that it is difficult to implement in practice. The KT-based method was proposed in [29], which first employs the KT to eliminate the linear RM, and then eliminates the residual RM and DFM by jointly searching the acceleration, jerk, and Doppler ambiguity number. This method can acquire similar target detection capability and parameter estimation precision to the GRFT method, but the focusing performance of the proposed method may deteriorate severely when the Doppler spectrum of a maneuvering target, whose bandwidth is greater than half of the pulse repetition frequency (PRF), is not entirely in one PRF band. Moreover, the computational burden of the KT-based method proposed in [29] is still heavy due to a 3D parameters search. In [30], Li et al. presented the generalized KT and generalized dechirp process (GKTGDP) algorithm to achieve target detection, which corrected the RM by performing high-order KT three times and searching the Doppler ambiguity number. However, this algorithm may suffer from great performance losses because it ignores the effect of Doppler spectrum ambiguity. Huang et al. proposed an algorithm based on SOKT and Hough-generalized high-order ambiguity function (SOKT-GHHAF) [31], which estimates the target's motion parameters by introducing high-order transform kernels and eliminating the Doppler spectrum ambiguity via a preprocessing step (shifting target Doppler center with half of the PRF). Nevertheless, the preprocessing is useless if the bandwidth of the target Doppler spectrum is greater than half of the PRF. The improved 2D matched filter algorithm [32] was presented to accumulate the weak target using a 2D grid search in the range-frequency and azimuth-Doppler domain. However, under the condition of a small time-bandwidth product, this algorithm may be inappropriate.

It is worth noting that the abovementioned methods [28-32] have a high computational burden due to multidimensional motion parameter search. To decrease the computational cost, several computationally efficient algorithms were recently presented. A method [33] based on time reversing transform, second-order keystone transform, and Lv distribution (TRT-SOKT-LVD) was developed to refocus the maneuvering only using a 1D parameter 
search. However, the bandwidth of the target spectrum is doubled, which may aggravate the Doppler spectrum ambiguity. The adjacent cross-correlation function iteratively (IACCF) method [34] was presented to accumulation the target energy via two ACCF operations. Although the IAACF can be quickly implemented via fast Fourier transform (FFT) and inverse FFT (IFFT), its integration performance is poor because the nonlinear transform is applied twice in the IACCF method. In [35], a fast algorithm based on auto-correlation function, polynomial-phase transform, and LVD (ACF-PPT-LVD) was developed to remove the RM and DFM by decreasing the phase order. Nevertheless, the application of a high-order nonlinear transform resulted in a serious deterioration of focusing performance.

Motivated by previous researches, we propose an efficient method for ground maneuvering target refocusing based on discrete polynomial-phase transform (DPT), KT, and matched filtering processing (MFP), i.e., DPT-KT-MFP. Firstly, the DPT is performed to simultaneously eliminate the quadratic RM, cubic RM, and quadratic DFM. Furthermore, the effects of Doppler ambiguity containing Doppler center blur and Doppler spectrum ambiguity are removed by introducing a very small fixed lay time after DPT operation. After that, the KT is applied to correct the linear RM. Then, a matched filtering function is constructed to compensate for the residual linear DFM caused by the equivalent third-order coefficient in the range-time and slow-time domain. After matching the proper equivalent third-order coefficient in the 1D parameter searching space and eliminating the effect of linear DFM, a well-refocused image of the maneuvering target can be acquired, and then the target's motion parameters can be effectively estimated. The contributions of this paper are as follows:

1. The presented DPT-KT-MFP method can effectively remove the high-order RM and DFM caused by a ground maneuvering target's complex motions.

2. The presented method can effectively deal with the Doppler ambiguity containing Doppler center blur and Doppler spectrum ambiguity.

3. The proposed algorithm has high compensational efficiency since only the target's equivalent third-order coefficient, which is usually very small for ground maneuvering targets, requires to be searched.

4. The proposed algorithm has high motion parameter estimation accuracy due to it avoiding the error propagation effect.

The remainder of this paper is organized as follows: in Section 2, the mathematical model of the received signal is established. In Section 3, the proposed refocusing algorithm is introduced. Section 4 presents some simulation experiments to evaluate the refocusing performance of the proposed method. At last, the work is concluded in Section 5.

\section{Signal Model}

The motion geometry relationship between the SAR platform with the constant speed $v$ and the ground moving target on the 2D slant plane is given in Figure 1, where the radar works in the side-looking mode. The maneuvering target moves from a to $\mathrm{b}$ within the synthetic aperture time $T_{a} . v_{c}, a_{c}, v_{a}$, and $a_{a}$ are the target's cross-track velocity, cross-track acceleration, along-track velocity, and along-track acceleration, respectively, whereas $R_{0}$ is the target's nearest slant range.

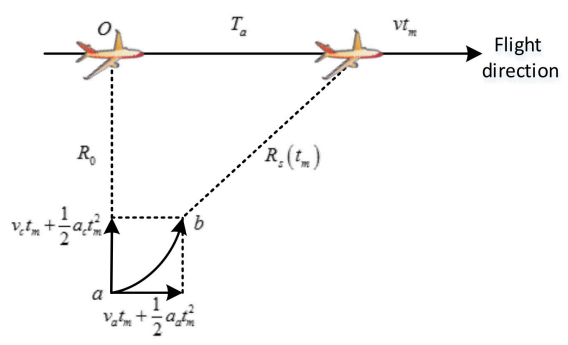

Figure 1. Motion geometry relationship between the synthetic aperture radar (SAR) platform and the ground moving target. 
The instantaneous slant range between the radar and the maneuvering target is described as follows [31]:

$$
R_{s}\left(t_{m}\right)=\sqrt{\left(v t_{m}-v_{a} t_{m}-\frac{1}{2} a_{a} t_{m}^{2}\right)^{2}+\left(R_{0}-v_{c} t_{m}-\frac{1}{2} a_{c} t_{m}^{2}\right)^{2}} \approx R_{0}+c_{1} t_{m}+c_{2} t_{m}^{2}+c_{3} t_{m}^{3},
$$

where $t_{m}=m T_{r}(m=-M / 2, \ldots, M / 2-1)$ represents the slow time, $M$ represents the integrated pulse number, $T_{r}$ denotes the pulse repetition time, and $c_{1}=-v_{c}, c_{2}=$ $\left(v-v_{a}\right)^{2} / 2 R_{0}-a_{c} / 2$, and $c_{3}=a_{a}\left(v_{a}-v\right) / 2 R_{0}+v_{c}\left(v-v_{a}\right)^{2} / 2 R_{0}^{2}$ denote the equivalent first-order coefficient, second-order coefficient, and third-order coefficient of $R_{s}\left(t_{m}\right)$, respectively.

Assuming that the radar transmits a linear frequency modulated (LFM) signal, the received baseband signal can be described as follows [33,34]:

$$
s_{r}\left(t, t_{m}\right)=A_{0} w_{a}\left(t_{m}\right) \operatorname{rect}\left(\frac{t-2 R_{s}\left(t_{m}\right) / c}{T_{p}}\right) \exp \left(-j \frac{4 \pi f_{c} R_{s}\left(t_{m}\right)}{c}\right) \exp \left(j \pi \mu\left(t-\frac{2 R_{s}\left(t_{m}\right)}{c}\right)^{2}\right),
$$

where $A_{0}$ is the maneuvering target's reflectivity, $\operatorname{rect}(\cdot)$ denotes the rectangle window function, $w_{a}\left(t_{m}\right)$ indicates the azimuth slow-time window function, and $t, T_{p}, f_{\mathcal{c}}, \mu$, and $c$ are the range time, pulse duration, carrier frequency, chirp rate, and speed of light, respectively.

After range compression, the received signal can be described as

$$
s_{1}\left(t, t_{m}\right)=A_{1} w_{a}\left(t_{m}\right) \operatorname{sinc}\left[B\left(t-\frac{2}{c}\left(R_{0}+c_{1} t_{m}+c_{2} t_{m}^{2}+c_{3} t_{m}^{3}\right)\right)\right] \exp \left[-j \frac{4 \pi}{\lambda}\left(R_{0}+c_{1} t_{m}+c_{2} t_{m}^{2}+c_{3} t_{m}^{3}\right)\right],
$$

where $A_{1}$ denotes the amplitude after range compression, whereas $B$ and $\lambda$ indicate the bandwidth and wavelength of transmitted signal, respectively. In general, because of the maneuvering target's high-speed motion and low PRF, there is a velocity ambiguity problem. Therefore, the target's equivalent speed can be described as

$$
c_{1}=n_{a m} v_{a}+v_{\text {base }}
$$

where $n_{a m}$ represents the Doppler ambiguity number, $v_{a}=\lambda f_{p r f} / 2$ denotes the blind velocity, $f_{\text {prf }}$ represents the PRF, and $v_{\text {base }}$ indicates the baseband velocity which satisfies $\left|v_{\text {base }}\right|<v_{a} / 2$.

Substituting Equation (4) into Equation (3) and performing range FFT yields

$$
\begin{aligned}
s_{1}\left(f, t_{m}\right)= & A_{2} \operatorname{rect}\left(\frac{f}{B}\right) w_{a}\left(t_{m}\right) \exp \left[-j \frac{4 \pi}{c}\left(f+f_{c}\right) n_{a m} v_{a} t_{m}\right] \\
& \times \exp \left[-j \frac{4 \pi}{c}\left(f+f_{c}\right)\left(R_{0}+v_{\text {base }} t_{m}+c_{2} t_{m}^{2}+c_{3} t_{m}^{3}\right),\right]
\end{aligned}
$$

where $A_{2}$ denotes the echo amplitude after range FFT, and $f$ denotes the range frequency variable corresponding to $t$.

Considering that $\exp \left(-j 4 \pi f_{c} n_{a m} v_{a} t_{m} / c\right)=1$, Equation (5) can be rewritten as

$$
\begin{aligned}
s_{1}\left(f, t_{m}\right) & =A_{2} \operatorname{rect}\left(\frac{f}{B}\right) w_{a}\left(t_{m}\right) \exp \left[-j \frac{4 \pi f}{c} n_{a m} v_{a} t_{m}\right] \\
& \times \exp \left[-j \frac{4 \pi}{c}\left(f+f_{c}\right)\left(R_{0}+v_{\text {base }} t_{m}+c_{2} t_{m}^{2}+c_{3} t_{m}^{3}\right)\right] .
\end{aligned}
$$

From Equation (6), we can find that the $t_{m}-$ term, $t_{m}^{2}-$ term, and $t_{m}^{3}-$ term are coupled with $f$, which may respectively result in the linear, quadratic, and cubic RM. Moreover, the $t_{m}$ - term, $t_{m}^{2}$ - term, and $t_{m}^{3}$ - term may induce the Doppler center shift, linear DFM, and quadratic DFM, respectively. Note that Doppler center blur occurs if the Doppler center shift is greater than $f_{p r f} / 2$. In addition, as shown in Figure 2, the target spectrum distributions can be classified as the following three cases: (1) target spectrum is overall in a PRF band [13]; (2) target spectrum occupies two neighboring PRF bands (target spectrum bandwidth is less than one PRF) [36]; (3) target spectrum spans over several 
PRF bands (target spectrum bandwidth is larger than one PRF) [37]. Obviously, the target Doppler spectrum is split in cases 2 and 3 since it is not overall in a PRF band, thereby bringing about Doppler spectrum ambiguity. To solve these problems, we develop an efficient method for ground maneuvering target refocusing based on DPT-KT-MFP in this paper.

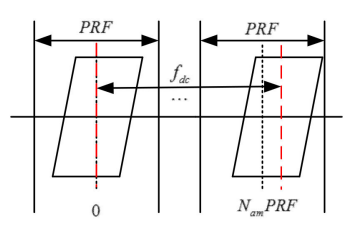

(a)

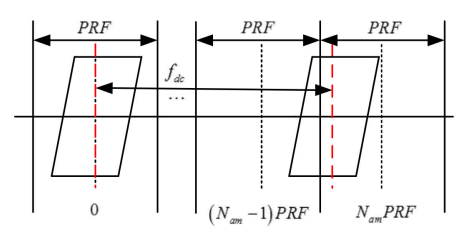

(b)

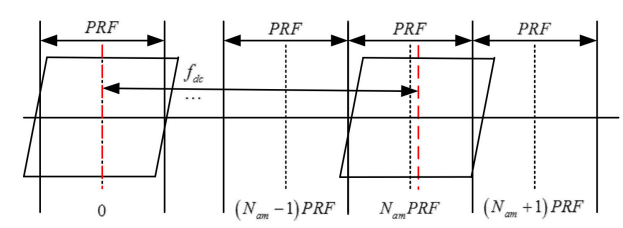

(c)

Figure 2. Target spectrum along azimuth. (a) Case 1: Target spectrum is entirely in one pulse repetition frequency (PRF) band. (b) Case 2: Target spectrum occupies two neighboring PRF bands. (c) Case 3: Target spectrum spans over several PRF bands.

\section{Algorithm Description}

\subsection{DPT-KT-MFP Algorithm with Single Target}

The DPT [38,39] can be applied to decrease the target phase order, which can be stated as

$$
s_{2}\left(f, t_{m}\right)=s_{1}\left(f, t_{m}+\frac{\tau_{0}}{2}\right) \cdot\left[s_{1}\left(f, t_{m}-\frac{\tau_{0}}{2}\right)\right]^{*},
$$

where $*$ is the conjugate operation, and $\tau_{0}$ is the fixed delay time, which is usually very small. Substituting Equation (6) into Equation (7) yields

$$
\begin{aligned}
s_{2}\left(f, t_{m}\right) & =A_{2}^{2} \operatorname{rect}\left(\frac{f}{B}\right) w_{a}\left(t_{m}\right) \exp \left(-j \frac{4 \pi n_{a m} v_{a} \tau_{0}}{\lambda} \frac{f}{f_{c}}\right) \\
& \times \exp \left(-j \frac{\pi\left(4 v_{\text {base }} \tau_{0}+c_{3} \tau_{0}^{3}\right)}{\lambda}\left(\frac{f+f_{c}}{f_{c}}\right)\right) \exp \left(-j \frac{4 \pi\left(2 c_{2} \tau_{0} t_{m}+3 c_{3} \tau_{0} t_{m}^{2}\right)}{\lambda}\left(\frac{f+f_{c}}{f_{c}}\right)\right) .
\end{aligned}
$$

From Equation (8), we can find that the order of $t_{m}$ is reduced to second order, which means that the quadratic DFM and cubic RM caused by the third-order coefficient $c_{3}$ is removed. Moreover, under the condition of $\left(v-v_{a}\right) T_{a} \ll R_{0}$, the quadratic RM caused by $c_{3}$ can be ignored since its maximum offset $3 c_{3} \tau_{0} T_{a}^{2} / 4$ is much smaller than a range resolution cell. Moreover, the Doppler center and Doppler spectrum bandwidth are changed to $4 c_{2} \tau_{0} / \lambda$ and $12 c_{3} \tau_{0} T_{a} / \lambda$, respectively. For a ground moving target, $c_{2}, c_{3}$, and $\tau_{0}$ are so small that the azimuth Doppler spectrum of the maneuvering target is entirely distributed in the non-ambiguous Doppler area. Thus, the problem of Doppler center blur and Doppler spectrum ambiguity is effectively addressed. However, the linear RM caused by $c_{2}$ and linear DFM induced by $c_{3}$ still exist after the DPT procedure, which can deteriorate the focusing performance.

In $[14,24,39]$, several algorithms were proposed to correct the RM related to secondorder coefficient $c_{2}$ by constructing a compensation function. However, constructing the compensation function needs some prior knowledge, such as platform velocity or the target's nearest slant range, which cannot always be accurately obtained. Additionally, the compensation error of RM caused by these methods [14,24,39] may be larger than a range resolution cell in the high-resolution case, which may result in a serious refocusing performance loss.

Consequently, in this paper, the KT was applied to absolutely remove the linear RM without any prior knowledge. The KT can be stated as follows [10-14,24,29]:

$$
t_{m}=\frac{f_{c}}{f+f_{c}} t_{n},
$$


where $t_{n}$ indicates the new slow-time variable. Substituting Equation (9) into Equation (8) yields

$$
\begin{aligned}
s_{2}\left(f, t_{n}\right) & =A_{2}^{2} \operatorname{rect}\left(\frac{f}{B}\right) w_{a}\left(t_{n}\right) \exp \left(-j \frac{4 \pi n_{a m} v_{a} \tau_{0}}{\lambda} \frac{f}{f_{c}}\right) \exp \left(-j \frac{8 \pi c_{2} \tau_{0} t_{n}}{\lambda}\right) \\
& \times \exp \left(-j \frac{12 \pi c_{3} \tau_{0} t_{n}^{2}}{\lambda}\left(\frac{f_{c}}{f+f_{c}}\right)\right) \exp \left(-j \frac{\pi\left(4 v_{\text {bass }} \tau_{0}+c_{3} \tau_{0}^{3}\right)}{\lambda}\left(\frac{f+f_{c}}{f_{c}}\right)\right) .
\end{aligned}
$$

Under the narrowband condition, $f_{c} /(f+f) \approx 1-f / f_{c}$, Equation (10) can be rewritten as

$$
\begin{aligned}
s_{2}\left(f, t_{n}\right) & =A_{2}^{2} \operatorname{rect}\left(\frac{f}{B}\right) w_{a}\left(t_{n}\right) \exp \left(-j \frac{4 \pi n_{a m} v_{a} \tau_{0}}{\lambda} \frac{f}{f_{c}}\right) \exp \left(-j \frac{8 \pi c_{2} \tau_{0} t_{n}}{\lambda}\right) \\
& \times \exp \left(-j \frac{12 \pi c_{3} \tau_{0} t_{n}^{2}}{\lambda}\left(1-\frac{f}{f_{c}}\right)\right) \exp \left(-j \frac{\pi\left(4 v_{\text {bass }} \tau_{0}+c_{3} \tau_{0}^{3}\right)}{\lambda}\left(\frac{f+f_{c}}{f_{c}}\right)\right) .
\end{aligned}
$$

From Equation (11), one can see that the coupling between the $t_{n}$ - term and $f$ is removed, which means that the linear RM is effectively corrected. Moreover, as for the ground maneuvering target, the $\exp \left(-j 12 \pi c_{3} \tau_{0} t_{n}^{2} f / c\right)$ can be ignored because its value is much smaller than $\pi / 4$ due to the small values of $c_{3}$ and $\tau_{0}$ [27].

After performing the range IFFT on Equation (11), we obtain

$$
\begin{aligned}
s_{3}\left(t, t_{n}\right)= & A_{3} w_{a}\left(t_{n}\right) \operatorname{sinc}\left\{t-\frac{2 c_{1} \tau_{0}}{c}-\frac{c_{3} \tau_{0}^{3}}{2 c}\right\} \exp \left(-j \frac{8 \pi c_{2} \tau_{0} t_{n}}{\lambda}\right) \\
& \times \exp \left(-j \frac{12 \pi c_{3} \tau_{0} t_{n}^{2}}{\lambda}\right) \exp \left(-j \frac{\pi}{\lambda}\left(4 v_{\text {base }} \tau_{0}+c_{3} \tau_{0}^{3}\right)\right),
\end{aligned}
$$

where $A_{3}$ represents the echo amplitude after IFFT. From Equation (12), we can find that the RM containing linear, quadratic, and cubic RM is effectively removed via the previous operation. However, due to the existence of the linear DFM caused by third-order coefficient $c_{3}$, the maneuvering target is still seriously defocused if we directly apply the azimuth FFT to accumulate the target energy. The previous methods proposed in $[24,31,33,39]$ assumed that the target signal is an LFM signal after correcting the RM, and they utilized timefrequency analysis algorithms to remove the linear DFM. Nevertheless, these methods need to search the range cells for extracting the target energy, which greatly increases the computational complexity. Actually, the maximum offset of linear DFM induced by $c_{3}$, which is equal to $12 c_{3} \tau_{0} T_{a} / \lambda$, is usually very small because of the small values of $c_{3}$ and $\tau_{0}$. The linear DFM induced by $c_{3}$ only spans across several Doppler frequency resolution cells. Therefore, the searching third-order coefficient is much smaller than the searching range cells. Hence, we constructed a matched filtering function to remove the linear DFM, which can be expressed as

$$
H\left(t, t_{n}\right)=\exp \left(j \frac{12 \pi a_{3} \tau_{0} t_{n}^{2}}{\lambda}\right)
$$

where $a_{3}=l \Delta a_{3}(l=-L / 2, \ldots, L / 2-1)$ is the searching third-order coefficient, $L$ denotes the size of the searching third-order coefficient, and $\Delta a_{3}$ is the searching step of $a_{3}$.

From Equation (14), we can find that the Doppler frequency related to $a_{3}$ is $12 a_{3} \tau_{0} t_{n} / \lambda$. The criterion for selecting $\Delta a_{3}$ is that the maximum offset of $12 \Delta a_{3} \tau_{0} t_{n} / \lambda$ is equal to a Doppler frequency resolution cell in one coherent process interval [23]. Therefore, the $\Delta a_{3}$ can be described as

$$
\Delta a_{3}=\frac{\lambda f_{p r f}^{2}}{12 M^{2} \tau_{0}}
$$

When $a_{3}$ is equal to $c_{3}$, multiplying Equation (13) by Equation (12) and performing the azimuth FFT yields 


$$
\begin{aligned}
s_{4}\left(t, f_{n}\right)= & \operatorname{FFT}_{t_{n}}\left[s_{3}\left(t, t_{n}\right) \times H\left(t, t_{n}\right)\right]=A_{4} \operatorname{sinc}\left\{t-\frac{2 c_{1} \tau_{0}}{c}-\frac{c_{3} \tau_{0}^{3}}{2 c}\right\} \\
& \times \operatorname{sinc}\left(f_{n}+\frac{4 c_{2} \tau_{0}}{\lambda}\right) \exp \left(-j \frac{4 \pi v_{\text {base }} \tau_{0}}{\lambda}\right) \exp \left(-j \frac{\pi c_{3} \tau_{0}^{3}}{\lambda}\right)
\end{aligned}
$$

where $\mathrm{FFT}_{t_{n}}[]$ denotes the FFT operation, $A_{4}$ is the signal amplitude after FFT operation, and $f_{n}$ indicates the Doppler frequency variable corresponding to $t_{n}$. From Equations (13) and (15), it can be seen that, when the third-order coefficient $c_{3}$ is matched exactly, the maneuvering target is well refocused at $t=2 c_{1} \tau_{0} / c+0.5 c_{3} \tau_{0}^{3} / c$ and $f_{n}=-4 c_{2} \tau_{0} / \lambda$. As a result, using the peak detection technique [40], $c_{1}, c_{2}$, and $c_{3}$ can be estimated as follows:

$$
\begin{gathered}
\hat{c}_{3}=\underset{a_{3}}{\operatorname{argmax}}\left|\operatorname{FFT}_{t_{n}}\left\{\operatorname{IFFT}_{f}\left[\operatorname{KT}\left[\operatorname{DPT}\left(s_{1}\left(f, t_{m}\right)\right)\right]\right] \times H\left(t, t_{n}\right)\right\}\right|, \\
\hat{c}_{1}=\frac{2 c \hat{t}-\hat{c}_{3} \tau_{0}^{3}}{4 \tau_{0}} \quad \hat{c}_{2}=-\frac{\hat{f}_{n} \lambda}{4 \tau_{0}} .
\end{gathered}
$$

According to the aforementioned analyses, we know that the presented algorithm can effectively remove the effects of Doppler center blur, Doppler spectrum ambiguity, high-order RM, and DFM, as well as refocus the maneuvering target. Figure 3 gives the flowchart of the presented method.

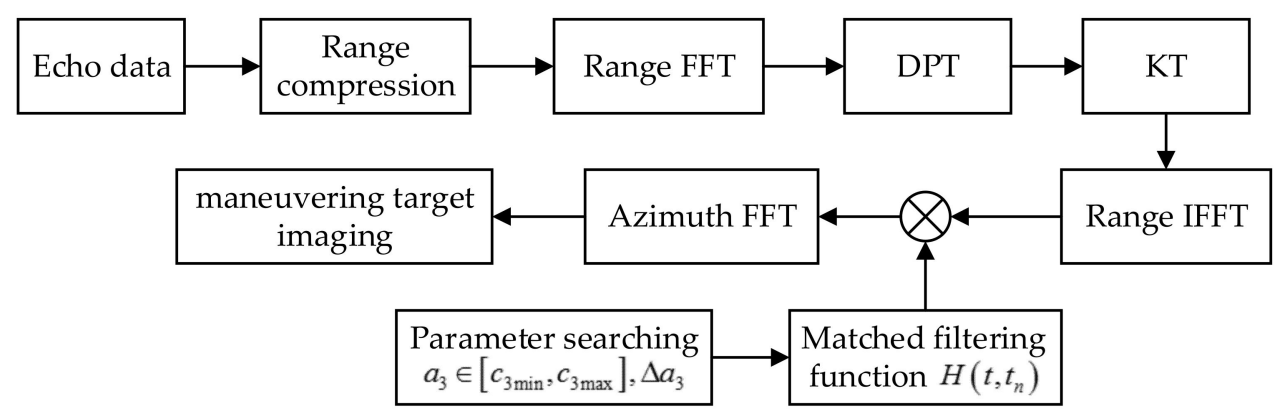

Figure 3. Processing steps of the presented discrete polynomial-phase transform (DPT), keystone transform (KT), and matched filtering processing (DPT-KT-MFP) algorithm.

Below, we give an example to demonstrate that the presented algorithm can effectively refocus a ground maneuvering target.

Example 1: Suppose a ground maneuvering target exists in the observation area. The radar system parameters are listed in Table 1 , and the motion parameters of maneuvering target are set as follows: the nearest slant range $R_{0}=3000 \mathrm{~m}$, the along-track velocity $v_{a}=23 \mathrm{~m} / \mathrm{s}$, the along-track acceleration $a_{a}=-4.5 \mathrm{~m} / \mathrm{s}^{2}$, the cross-track velocity $v_{c}=32 \mathrm{~m} / \mathrm{s}$, and the cross-track acceleration $a_{c}=-3.6 \mathrm{~m} / \mathrm{s}^{2}$. According to Equation (1), the first-, second-, and third-order coefficients can be calculated as $c_{1}=-32 \mathrm{~m} / \mathrm{s}, c_{2}=$ $10.3882 \mathrm{~m} / \mathrm{s}^{2}$, and $c_{3}=0.2619 \mathrm{~m} / \mathrm{s}^{3}$. The simulation results are given in Figure 4 .

Table 1. System parameters of radar.

\begin{tabular}{cc}
\hline Parameter & Value \\
\hline Carrier frequency & $6 \mathrm{GH}_{Z}$ \\
Range bandwidth & $200 \mathrm{MH}_{\mathrm{Z}}$ \\
Range sampling frequency & $300 \mathrm{MHZ}_{Z}$ \\
Pulse repetition frequency & $800 \mathrm{H}_{\mathrm{Z}}$ \\
Pulse width & $1 \mu \mathrm{s}$ \\
Synthetic aperture time & $2 \mathrm{~s}$ \\
Platform velocity & $250 \mathrm{~m} / \mathrm{s}$ \\
\hline
\end{tabular}

Figure 4a gives the range compression result. Obviously, serious RM containing linear, quadratic, and cubic RM occurs. The target Doppler spectrum is given in Figure $4 \mathrm{~b}$. It 
can be seen that the target spectrum occupies three PRF bands, as shown in Figure 2c, which means that serious Doppler spectrum ambiguity appears. The target trajectory after DPT operation is shown in Figure 4c, from which we can find that an oblique straight line is present, which means that the quadratic and cubic RM is effectively removed. The target spectrum after DPT operation is given in Figure 4d. We can observe that the target spectrum is overall distributed in one PRF band, which means that the problem of Doppler spectrum ambiguity is addressed. Additionally, the Doppler center blur is also eliminated after DPT operation, since the Doppler center is changed from $320 \mathrm{H}_{Z}\left(n_{a m}=-2\right)$ to $166.2 \mathrm{H}_{\mathrm{Z}}\left(n_{a m}=0\right)$. Figure 4e shows the result after KT operation. We can find that target trajectory becomes one straight line along the slow-time axis, which means that the residual linear RM is effectively corrected. Figure $4 \mathrm{f}$ shows the searching result of the third-order coefficient. Obviously, according to the peak position, $c_{3}$ can be estimated as $0.2624 \mathrm{~m} / \mathrm{s}^{3}$. After correcting the linear DFM using the estimated third-order coefficient, we can observe that a distinct peak appears in Figure $4 \mathrm{~g}$, which indicates that the presented algorithm can effectively remove the Doppler center blur, Doppler spectrum ambiguity, high-order RM, and DFM, as well as refocus the maneuvering target. On the basis of peak detection technology [40], the first- and second-order coefficients can be estimated as follows: $\hat{c}_{1}=32.0013 \mathrm{~m} / \mathrm{s}$ and $\hat{c}_{2}=10.3875 \mathrm{~m} / \mathrm{s}^{2}$.

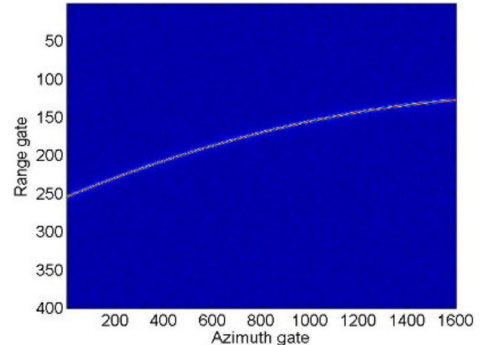

(a)

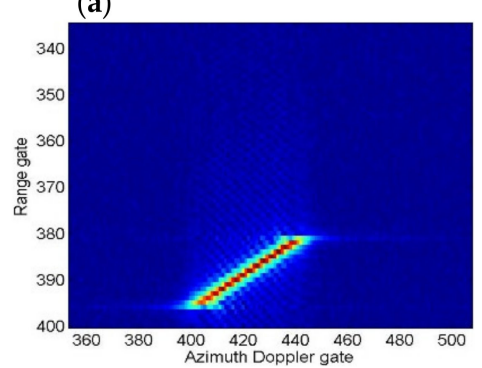

(d)

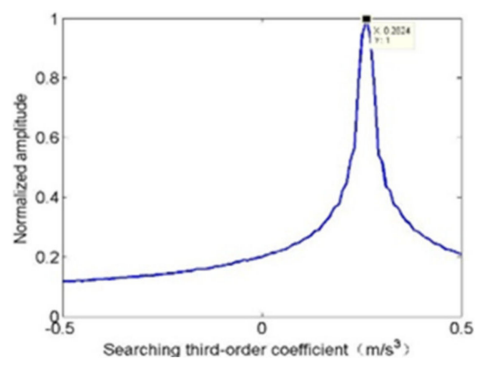

(f)

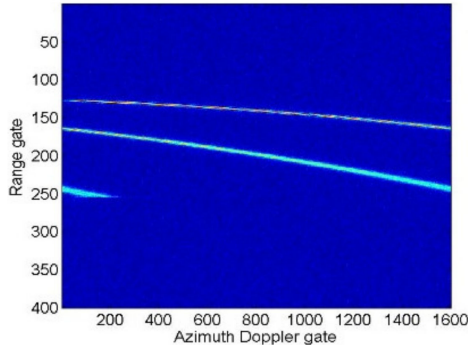

(b)

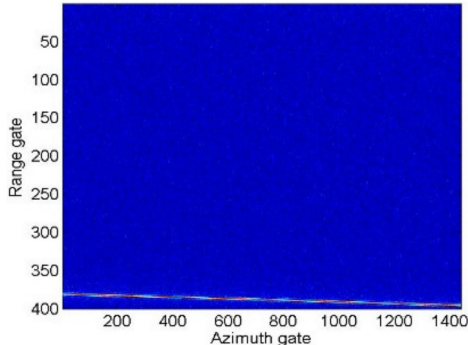

(c)

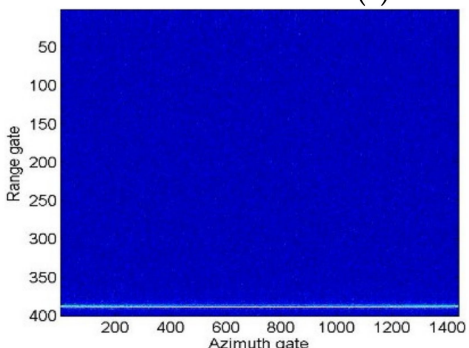

(e)

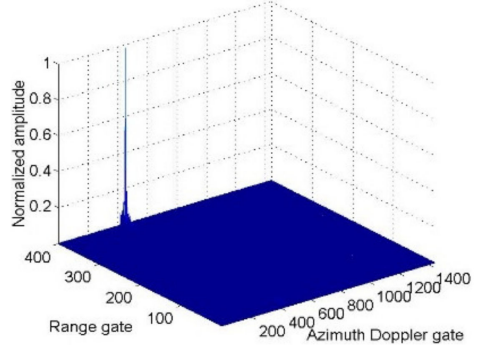

(g)

Figure 4. Simulation results of Example 1. (a) Target trajectory after range compression. (b) Corresponding target Doppler spectrum. (c) Target trajectory after DPT. (d) Corresponding target Doppler spectrum after DPT. (e) Result after KT. (f) Searching result of third-order coefficient. (g) Focusing result of the proposed algorithm.

\subsection{DPT-KT-MFP Algorithm with Multiple Targets}

With respect to refocusing multiple maneuvering targets, cross-term interference is inevitably introduced because of the existence of nonlinear transform in our proposed 
method. In this subsection, the effects of cross-terms are analyzed. Suppose that $K$ maneuvering targets exist in the observation area; then, the range compression signal in $f-t_{m}$ domain can be stated as

$$
\begin{aligned}
& s_{m u l, 1}\left(f, t_{m}\right)=\sum_{i=1}^{K} A_{2, i} \operatorname{rect}\left(\frac{f}{B}\right) w_{a}\left(t_{m}\right) \exp \left[-j \frac{4 \pi R_{0, i}}{c}\left(f+f_{c}\right)\right] \\
& \times \exp \left(-j \frac{4 \pi f}{c} n_{a m, i} v_{a} t_{m}\right) \exp \left[-j \frac{4 \pi}{c}\left(f+f_{c}\right)\left(v_{\text {base }, i} t_{m}+c_{2, i} t_{m}^{2}+c_{3, i} t_{m}^{3}\right)\right],
\end{aligned}
$$

where $A_{2, i}$ represents the $i$ th target's amplitude, and $R_{0, i}$ denotes the $i$ th target's nearest slant range. $n_{a m, i}, v_{b a s e, i}, c_{2, i}$, and $c_{3, i}$ represent the Doppler ambiguity number, baseband velocity, second- and third-order coefficients of the $i$ th moving target, respectively.

After performing the DPT, we obtain

$$
\begin{aligned}
& s_{m u l, 1}\left(f, t_{m}\right)=\sum_{i=1}^{K} A_{2, i}^{2} \operatorname{rect}\left(\frac{f}{B}\right) w_{a}\left(t_{m}\right) \exp \left[-j \frac{\pi\left(4 v_{0, i} \tau_{0}+c_{3, i} \tau_{0}^{3}\right)}{\lambda}\left(\frac{f+f_{c}}{f_{c}}\right)\right] \\
& \times \exp \left[-j \frac{4 \pi\left(2 c_{2, i} t_{m}+3 c_{3, i} t_{m}^{2}\right) \tau_{0}}{\lambda}\left(\frac{f+f_{c}}{f_{c}}\right)\right] \exp \left(-j \frac{4 \pi n_{a m, i} v_{a} \tau_{0}}{\lambda} \frac{f}{f_{c}}\right)+R_{\text {cross }}\left(f, t_{m}\right),
\end{aligned}
$$

where

$$
\begin{aligned}
& R_{\text {cross }}\left(f, t_{m}\right)=\sum_{i=1}^{K} \sum_{j=1, i \neq j}^{K} A_{2, i} A_{2, j} \operatorname{rect}\left(\frac{f}{B}\right) w_{a}\left(t_{m}\right) \exp \left[-j \frac{4 \pi}{\lambda}\left(\frac{f+f_{c}}{f_{c}}\right)\left(R_{0, i}-R_{0, j}\right)\right] \\
& \times \exp \left[-j \frac{4 \pi v_{a} t_{m}}{\lambda} \frac{f}{f_{c}}\left(n_{a m, i}-n_{a m, j}\right)\right] \exp \left[-j \frac{2 \pi v_{a} \tau_{0}}{\lambda} \frac{f}{f_{c}}\left(n_{a m, i}+n_{a m, j}\right)\right] \\
& \times \exp \left[-j \frac{4 \pi}{\lambda}\left(\frac{f+f_{c}}{f_{c}}\right)\left(v_{0, i}-v_{0, j}\right) t_{m}\right] \exp \left[-j \frac{2 \pi}{\lambda}\left(\frac{f+f_{c}}{f_{c}}\right)\left(v_{0, i}+v_{0, j}\right) \tau_{0}\right] \\
& \times \exp \left[-j \frac{\pi}{\lambda}\left(\frac{f+f_{c}}{f_{c}}\right)\left(c_{2, i}-c_{2, j}\right)\left(4 t_{m}^{2}+\tau_{0}^{2}\right)\right] \exp \left(-j \frac{4 \pi}{\lambda}\left(\frac{f+f_{c}}{f_{c}}\right)\left(c_{2, i}+c_{2, j}\right) t_{m} \tau_{0}\right) \\
& \times \exp \left[-j \frac{\pi}{\lambda}\left(\frac{f+f_{c}}{f_{c}}\right)\left(c_{3, i}-c_{3, j}\right)\left(4 t_{m}^{3}+3 t_{m} \tau_{0}^{2}\right)\right] \exp \left[-j \frac{\pi}{\lambda}\left(\frac{f+f_{c}}{f_{c}}\right)\left(c_{3, i}+c_{3, j}\right)\left(6 t_{m}^{2} \tau_{0}+0.5 \tau_{0}^{3}\right)\right] .
\end{aligned}
$$

From Equations (19) and (20), it can be seen that, with respect to the auto-terms after DPT, the cubic RM and quadratic DFM are effectively removed. Furthermore, similar to refocusing the single target, the quadratic RM caused by $c_{3, i}$ can be ignored since its maximum offset $3 c_{3, i} \tau_{0} T_{a}^{2} / 4$ is much smaller than a range resolution cell. For the crossterms after DPT, the high-order RM and DFM are still present, because the $t_{m}-$ term, $t_{m}^{2}-$ term, and $t_{m}^{3}-$ term are still coupled with $f$. Consequently, after carrying out the KT and MFP operations as described in Section 3.1, the energy of the auto-terms is well coherently accumulated, while the energy of the cross-terms cannot be integrated effectively because of the high-order RM and DFM. In addition, the proposed method can also refocus the maneuvering targets in the case of close multiple targets, since the $R_{0, i}$ in auto-terms is effectively removed after DPT operation. On the basis of the aforementioned analyses, we can conclude that the presented method is able to remove the effects of cross-terms and refocus the ground maneuvering targets in the case of multiple targets. Next, several simulation experiments are presented to validate how the presented method suppresses the cross-terms and focuses the auto-terms.

Example 2: Assume two maneuvering targets, represented by T1 and T2, exist in the observation area. The radar system parameters are the same as those in Example 1. In addition, the motion parameters of the two maneuvering targets are set as follows: $R_{0,1}=2940 \mathrm{~m}, v_{a, 1}=11 \mathrm{~m} / \mathrm{s}, a_{a, 1}=-4.5 \mathrm{~m} / \mathrm{s}^{2}, v_{c, 1}=32 \mathrm{~m} / \mathrm{s}$, and $a_{c, 1}=-3.6 \mathrm{~m} / \mathrm{s}^{2}$ for $\mathrm{T} 1 ; R_{0,2}=3050 \mathrm{~m}, v_{a, 2}=13 \mathrm{~m} / \mathrm{s}, a_{a, 2}=4.3 \mathrm{~m} / \mathrm{s}^{2}, v_{c, 2}=-36 \mathrm{~m} / \mathrm{s}$, and $a_{c, 2}=-1.5 \mathrm{~m} / \mathrm{s}^{2}$ for T2. On the basis of Equation (1), the first-, second-, and third-order coefficients of T1 and T2 can be calculated as follows: $c_{1,1}=-32, c_{2,1}=11.5145$, and $c_{3,1}=0.2886$ for T1; $c_{1,2}=36, c_{2,2}=9.9580$, and $c_{3,2}=-0.2758$ for T2. Figure 5 gives the simulation results.

Figure 5 a gives the target trajectories after range compression. Obviously, the targets' energy spans across several range cells during the coherent integration time. After DPT 
and KT operations, the RM correction result is given in Figure 5b, from which it can be seen that the energy of T1 and T2 is concentrated in the same range cells, while the energy of cross-terms still spans across several range cells. Figure $5 c$ shows the searching result of the third-order coefficient. It can be seen that two distinct peaks exist. According to the peak positions, the third-order coefficients of T1 and T2 can be estimated as follows: $\hat{c}_{3,1}=0.2879$ and $\hat{c}_{3,2}=-0.2778$. After compensating for the linear DFM using the estimated thirdorder coefficients, Figure 5d,e give the final focusing results of T1 and T2, respectively. Obviously, two well-focused peaks appear in Figure 5d,e, which demonstrates that the propose algorithm can effectively eliminate the effects of cross-terms and integrate the energy of auto-terms. According to the peak position, the remaining motion parameters of T1 and T2 can be estimated as follows: $\hat{c}_{1,1}=-31.9975$ and $\hat{c}_{2,1}=11.5162$ for T1; $\hat{c}_{1,2}=36.0019$ and $\hat{c}_{2,2}=9.9589$ for $\mathrm{T} 2$.

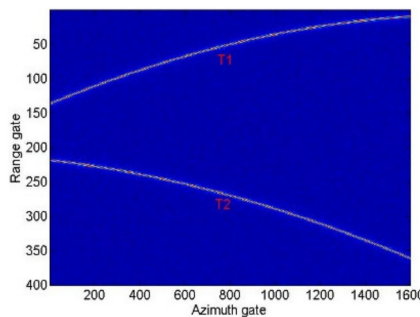

(a)

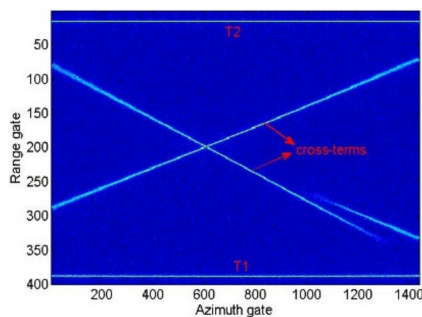

(b)

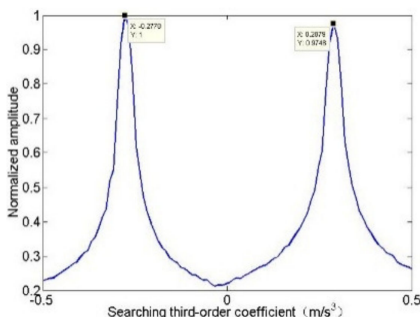

(c)

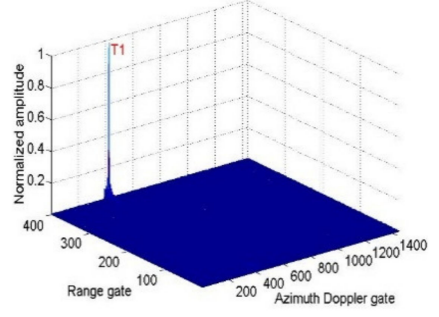

(d)

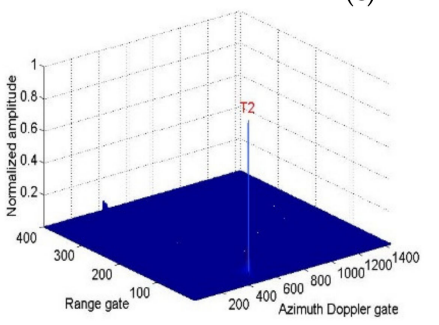

(e)

Figure 5. Simulation results of Example 2. (a) Target trajectories after range compression. (b) Result after RM correction. (c) Searching result of third-order coefficient. (d) Final focusing result of T1. (e) Final focusing result of T2.

\subsection{Computational Complexity Analysis}

Next, the computational cost of GRFT [28], SOKT-GHHAF [31], TRT-SOKT-LVD [33], and the proposed method was analyzed. Suppose $N, M$, and $M_{\tau}$ represent the numbers of range cells, echo pulses, and lag samples, respectively, and the number of searching first-, second-, and third-order coefficients is the same, denoted as $N_{1}$. The computational complexity of GRFT based on $4 \mathrm{D}$ brute-force search is approximately $\mathrm{O}\left(N_{1}^{3} N M\right)$. The procedures of the SOKT-GHHAF mainly include a SOKT operation, whose computational complexity is approximately $\mathrm{NMO}\left(\log _{2} M\right)$, a Hough transform (HT) operation, with a computational cost of $\mathrm{O}\left(N M^{2}\right)$, a GHHAF operation, whose computational complexity is approximately $\mathrm{O}\left(3 M^{2}+M^{3}+N_{1} M \log _{2} M\right)$, and a range cell search operation. Consequently, the total computational cost of SOKT-GHHAF is approximately $N M O\left(\log _{2} M\right)+\mathrm{O}\left(N M^{2}\right)+N O\left(3 M^{2}+M^{3}+N_{1} M \log _{2} M\right)$. The procedures of the TRTSOKT-LVD mainly include a TRT operation, whose computational cost is approximately $\mathrm{O}(N M)$, an SOKT operation with a computational complexity of $N M O\left(\log _{2} M\right)$, an LVD operation, whose computational complexity is approximately $M M_{\tau} \mathrm{O}\left(5 \log _{2} M+\log _{2} M_{\tau}\right)$, and a range cell search operation. As a consequence, the total computational cost of TRTSOKT-LVD is approximately $\mathrm{O}(N M)+N M \mathrm{O}\left(\log _{2} M\right)+N M M_{\tau} \mathrm{O}\left(5 \log _{2} M+\log _{2} M_{\tau}\right)$. In addition, the procedures of the proposed algorithm mainly include a DPT operation with a computational cost of $\mathrm{O}(N M)$, a KT operation with a computational complexity of $N M O\left(\log _{2} M\right)$, and an MFP operation, whose computational cost is approximately $N_{1} N M O\left(\log _{2} M\right)$. Therefore, the total computational cost of the proposed method is ap- 
proximately $\mathrm{O}(N M)+\left(N M+N_{1} N M\right) \mathrm{O}\left(\log _{2} M\right)$. The computational complexity of the abovementioned four algorithms is listed in Table 2.

Assume $N=M=M_{\tau}=N_{1}$; then, the computational costs of GRFT, SOKTGHHAF, TRT-SOKT-LVD, and the presented method are approximately $\mathrm{O}\left(N^{5}\right), \mathrm{O}\left(N^{4}\right)$, $\mathrm{O}\left(N^{3} \log _{2} N\right)$, and $\mathrm{O}\left(N^{3} \log _{2} N\right)$, respectively. Obviously, compared with GRFT and SOKT-GHHAF, the presented method has a much lower computational burden. Compared with the TRT-SOKT-LVD, the proposed algorithm has a similar computational burden under the above-assumed condition. Actually, with respect to the ground maneuvering target, the number of searching range cells $N$ is usually far greater than the number of searching third-order coefficients $N_{1}$. Consequently, the presented algorithm has a higher computational efficiency than the TRT-SOKT-LVD algorithm.

Below, the computational time of these four methods is compared, where the target's motion parameters and radar parameters are the same as those in Example 1 (the processor of the computer was a Intel (R) Core (TM) i7-4790 central processing unit (CPU) @3.60 $\mathrm{GHz}$; the random-access memory (RAM) was $8 \mathrm{~GB}$; the software was MATLAB 2014a). Table 3 shows the computational time of these methods, from which it can be seen that the presented algorithm is more efficient in comparison with the other three algorithms.

Table 2. Comparison of computational complexity. GRFT, generalized radon Fourier transform; SOKT-GHHAF, second-order KT and Hough-generalized high-order ambiguity function; TRTSOKT-LVD, time reversing transform, second-order KT, and Lv distribution.

\begin{tabular}{cc}
\hline Methods & Computational Complexity \\
\hline GRFT & $\mathrm{O}\left(N_{1}^{3} N M\right)$ \\
SOKT-GHHAF & $N M O\left(\log _{2} M\right)+\mathrm{O}\left(N M^{2}\right)+N O\left(3 M^{2}+M^{3}+N_{1} M \log _{2} M\right)$ \\
TRT-SOKT-LVD & $\mathrm{O}(N M)+N M O\left(\log _{2} M\right)+N M M_{\tau} \mathrm{O}\left(5 \log _{2} M+\log _{2} M_{\tau}\right)$ \\
Proposed & $\mathrm{O}(N M)+\left(N M+N_{1} N M\right) \mathrm{O}\left(\log _{2} M\right)$ \\
\hline
\end{tabular}

Table 3. Comparison of computational time.

\begin{tabular}{cc}
\hline Methods & Computational Time (s) \\
\hline GRFT & 3382 \\
SOKT-GHHAF & 279.2 \\
TRT-SOKT-LVD & 36.5 \\
Proposed & 3.49 \\
\hline
\end{tabular}

\section{Simulation and Real Data Processing Results}

\subsection{Analysis and Discussion of Simulation Results}

In this subsection, we adopt a simulated ideal point target to validate the effectiveness of the presented DPT-KT-MFP algorithm under the Gaussian noise environment. The target's motion parameters and radar parameters are the same as those in Example 1. In order to better show the superior performance of the presented DPT-KT-MFP algorithm, GRFT [28], SOKT-GHHAF [31], TRT-SOKT-LVD [33], and moving target detection (MTD) are performed for comparison.

\subsubsection{Maneuvering Target Integration Performance}

In Figure 6, we first evaluate the integration performance of the presented algorithm for a maneuvering target. The signal-to-noise ratio (SNR) after range compression was set as $6 \mathrm{~dB}$. It is worth noting that, on the basis of the target's motion parameters and radar system parameters set above, Doppler ambiguity (containing Doppler center blur and Doppler spectrum ambiguity) occurs. The target trajectory after range compression is shown in Figure 6a, from which we can find that the target energy is distributed in multiple range cells. Figure $6 \mathrm{~b}$ gives the direct KT result. One can see that the trajectory of the maneuvering target is split into three parts since its Doppler spectrum occupies three PRF bands. Figure $6 c$ gives the result after DPT and KT, from which noise is removed 
to clearly show the target trajectory. We can observe that the target energy is distributed in one range cell, since the RM, Doppler center blur, and Doppler spectrum ambiguity are eliminated after DPT and KT operations. The refocusing result of the presented DPTKT-MFP algorithm is given in Figure 6d. It can be seen that the target energy is well coherently integrated as a distinct peak. Figure 6e gives the MTD result. One can see that the MTD method cannot effectively accumulate the target energy because of the highorder RM and DFM caused by the complex motions of the maneuvering target. Figure $6 \mathrm{f}$ gives the focusing result of SOKT-GHHAF. Obviously, the SOKT-GHHAF method is invalid because of the Doppler spectrum ambiguity and the application of the incoherent integration algorithm. The result after TRT and SOKT is shown in Figure 6g. Similar to Figure $6 c$, the noise is removed to clearly show the target trajectory. We can observe that the target trajectory splits into five parts since the bandwidth of the target Doppler spectrum is doubled after TRT. Therefore, only a small fraction of target energy is accumulated by TRT-SOKT-LVD. Figure $6 \mathrm{~h}$ gives the TRT-SOKT-LVD result. Obviously, the maneuvering target is still seriously defocused due to the effects of Doppler spectrum ambiguity. The GRFT result is shown in Figure 6i. We can observe that the integration performance of the GRFT algorithm is better than the presented algorithm. Nevertheless, the good accumulation performance is at the cost of a high computational burden.

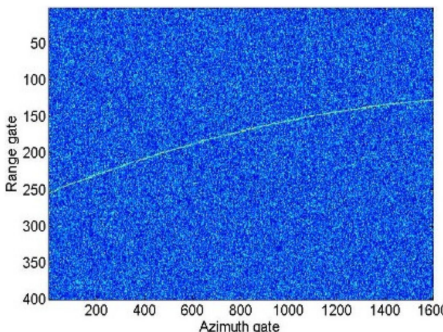

(a)

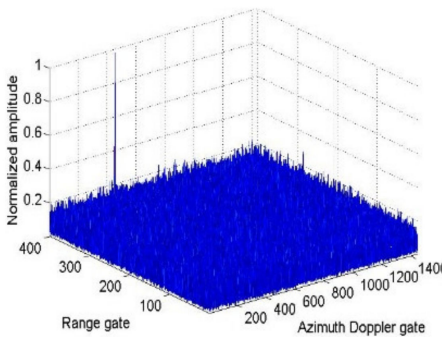

(d)

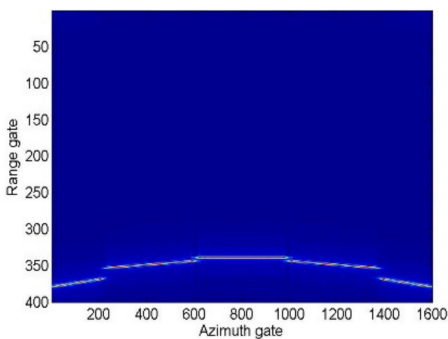

(g)

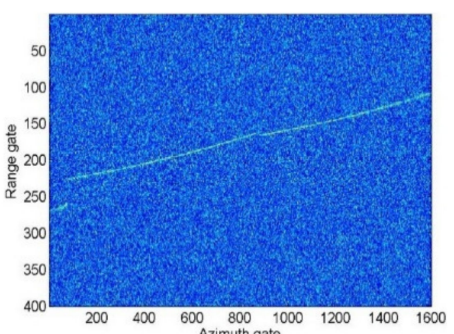

(b)

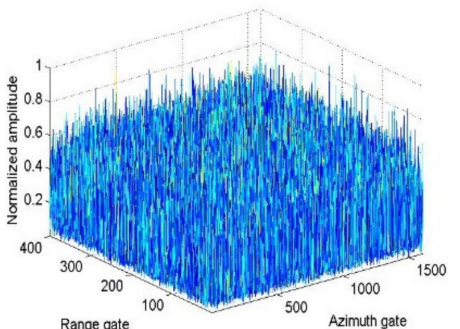

(e)

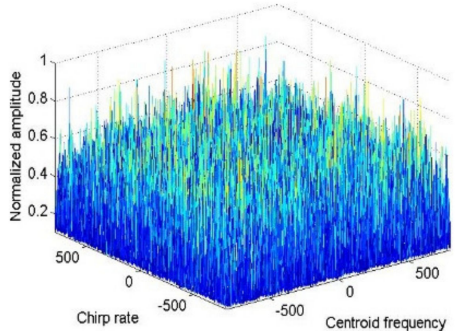

(h)

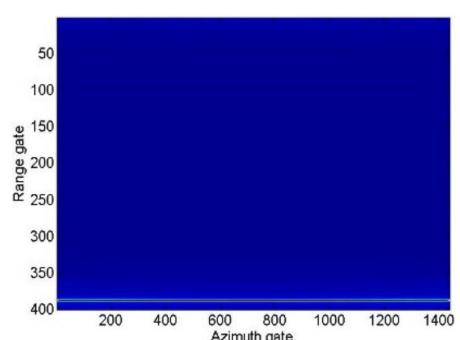

(c)

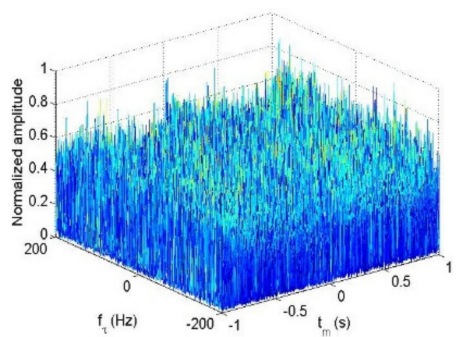

(f)

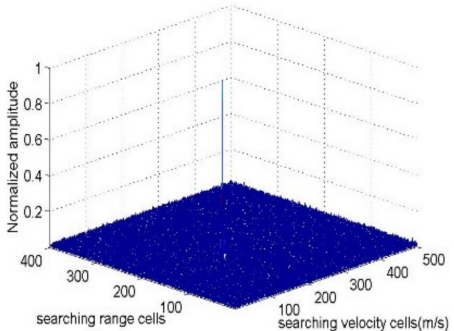

(i)

Figure 6. Refocusing results of different algorithms. (a) Range compression result. (b) Direct KT result. (c) Result after DPT and KT. (d) Focusing result of the presented algorithm. (e) Focusing result of MTD. (f) Focusing result of SOKT-GHHAF. (g) Result after TRT and SOKT. (h) Focusing result of TRT-SOKT-LVD. (i) Focusing result of GRFT. 


\subsubsection{Maneuvering Target Detection Ability}

Below, we analyze the detection ability of MTD, SOKT-GHHAF, TRT-SOKT-LVD, GRFT, and the proposed method via Monte Carlo trials. The constant false alarm probability was set at $10^{-6}$. After performing 500 Monte Carlo trials at every input SNR, Figure 7 shows the curves of detection probability varying with range compression SNRs. Obviously, the target detection performance of the presented algorithm was superior to that of the MTD, SOKT-GHHAF, and TRT-SOKT-LVD methods, since the effects of high-order RM and DFM, Doppler center blur, and Doppler spectrum ambiguity are effectively eliminated by the proposed algorithm. Compared with the GRFT method, the presented algorithm has a similar target detection ability but with a much lower computational cost when the input SNR is larger than 0dB. Unfortunately, the proposed method may suffer from a great detection performance loss in cases with low SNR, since the DPT is a nonlinear transform.

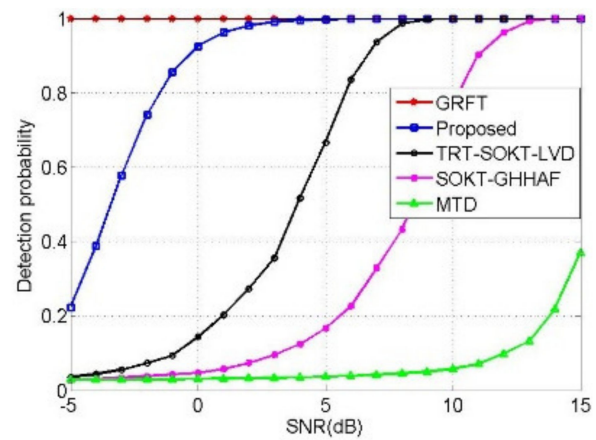

Figure 7. Curves of detection probability varying with range compression signal-to-noise ratios (SNRs).

\subsubsection{Motion Parameter Estimation Performance}

Next, the motion parameter estimation performance of SOKT-GHHAF, TRT-SOKTLVD, GRFT, and the presented algorithm was also compared via Monte Carlo trials, in which the input range-compressed SNRs varied from $-15 \mathrm{~dB}$ to $5 \mathrm{~dB}$ with a step size of $1 \mathrm{~dB}$. Moreover, at every input SNR, we carry out 500 Monte Carlo trials. Figure 8a-c show the root-mean-square errors (RMSEs) of the estimated first-, second-, and thirdorder coefficients varying with input SNRs, respectively. From Figure 8, one can see that the motion parameter estimation performance of SOKT-GHHAF method deteriorates severely, since the effects of Doppler ambiguity and cubic RM are ignored, and the HT, which is the incoherent integration operation, is used twice in the SOKT-GHHAF method. Moreover, SOKT-GHHAF has a huge computational burden due to the application of three 1D parameter searches. The TRT-SOKT-LVD method suffers from a great performance loss of parameter estimation, because it cannot remove the Doppler spectrum ambiguity. In comparison with the other three algorithms, the GRFT method can acquire the optimal motion parameter estimation performance, but it needs a $4 \mathrm{D}$ parameter search, which makes it difficult to implement in practice. When the input SNR is larger than 0dB, the motion parameter estimation performance of the presented algorithm is similar to that of the GRFT algorithm. Moreover, compared with the GRFT algorithm, the presented algorithm has a higher computational efficiency, since it only needs to search the thirdorder coefficient, whose value is usually very small for ground maneuvering targets. 


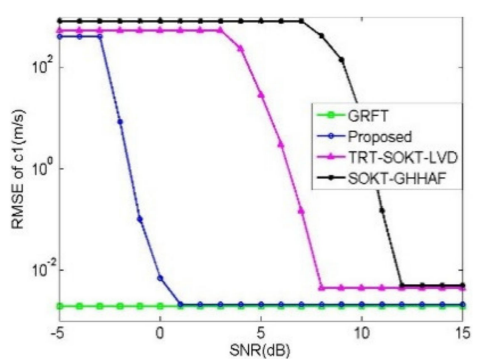

(a)

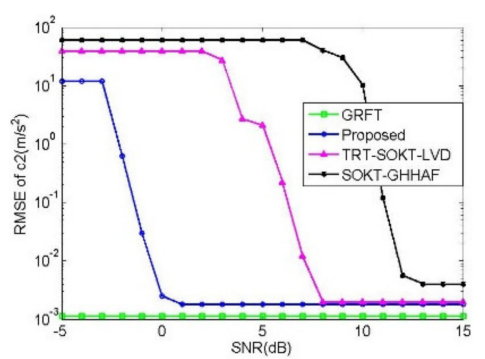

(b)

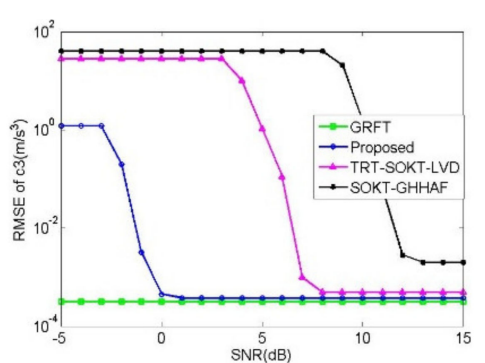

(c)

Figure 8. Relationships between root-mean-square errors (RMSEs) of motion parameters and input SNRs. (a) RMSE of estimated first-order coefficient varying with input SNRs. (b) RMSE of estimated second-order coefficient varying with input SNRs. (c) RMSE of estimated third-order coefficient varying with input SNRs.

\subsection{Analysis and Discussion of Real Data Processing Results}

In this subsection, we verify the effectiveness of the presented refocusing algorithm using real radar data recorded by an X-band airborne radar with a broadside mode. This radar has three apertures in the along-track direction, whereby one aperture transmits the signals and all three apertures receive the reflected echoes. The observed targets were cooperative moving trucks driving along the road. The SAR system parameters are listed in Table 4.

Figure 9a gives the image of the ground maneuvering targets. We can observe that the maneuvering targets are submerged by strong ground clutter. In order to obtain a higher target signal-to-clutter-plus-noise-ratio (SCNR), we carried out the extended factored algorithm [41] to suppress the ground clutter. After clutter suppression, Figure $9 \mathrm{~b}$ shows that several clear targets appear. However, the maneuvering targets are seriously smeared because of the RM and DFM. Below, we utilize a maneuvering target of interest marked by a red ellipse to demonstrate the effectiveness of the presented algorithm. The range compression result of the interested target is given in Figure 9c, from which we can find that the interested target energy is distributed in multiple range cells because of severe RM. The target trajectory after DPT and KT is shown in Figure $9 \mathrm{~d}$. it can be seen that the trajectory of the maneuvering target is parallel to the slow-time axis, which means that the RM is effectively eliminated. Figure 9e gives the focusing result of the presented algorithm. We can observe that a well-refocused peak appears since the high-order RM and DFM are absolutely removed, which proves the effectiveness of the presented DPT-KT-MFP algorithm.

Table 4. X-band SAR system parameters.

\begin{tabular}{cc}
\hline Parameter & Value \\
\hline Carrier frequency & $8.85 \mathrm{GHz}$ \\
Range bandwidth & $40 \mathrm{MHz}$ \\
Range sampling frequency & $60 \mathrm{MHz}$ \\
Pulse repetition frequency & $1000 \mathrm{~Hz}$ \\
Pulse width & $10 \mu \mathrm{s}$ \\
Platform velocity & $120 \mathrm{~m} / \mathrm{s}$ \\
Swath central range & $9000 \mathrm{~m}$ \\
\hline
\end{tabular}




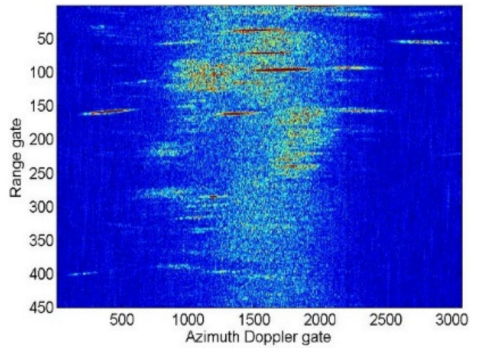

(a)

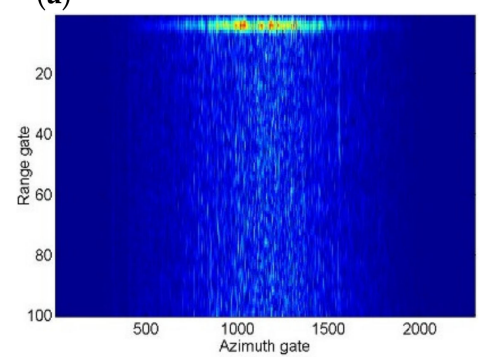

(d)

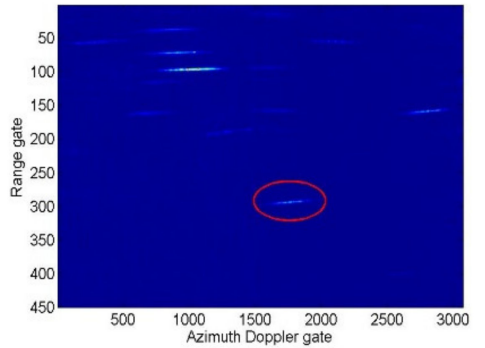

(b)

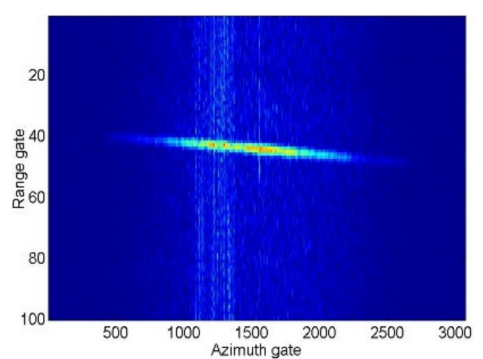

(c)

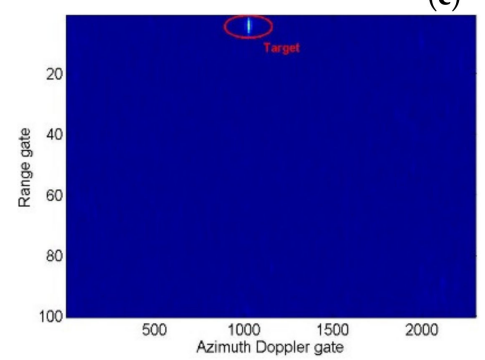

(e)

Figure 9. Refocusing results for a ground maneuvering target in a real SAR system. (a) Imaging result of ground maneuvering targets before clutter suppression. (b) Result after clutter suppression. (c) Moving trajectory of an interested target. (d) Result after DPT and KT operations. (e) Focusing result of the presented algorithm.

\section{Conclusions}

In this paper, the DPT-KT-MFP algorithm was presented to address the refocusing and motion parameter estimation problems for ground maneuvering targets, involving Doppler ambiguity, high-order RM, and DFM during the coherent integration time. Obviously, the proposed algorithm can effectively remove the high-order RM and DFM induced by complex motions of ground maneuvering targets, as well as realize the target refocusing with low computational complexity. In addition, the Doppler ambiguity containing Doppler center blur and Doppler spectrum ambiguity can also be effectively dealt with due to the DPT operation. Furthermore, for refocusing of multiple targets, the presented algorithm is able to eliminate the effects of cross-terms in which high-order RM and DFM still exist. Compared with conventional algorithms which ignore the effects of Doppler ambiguity and the target's high-order motions, the presented algorithm can acquire better focusing performance and motion parameter estimation precision. Furthermore, compared with the GRFT method, the presented algorithm has higher computational efficiency and similar focusing performance in the case of a high SNR.

Author Contributions: Conceptualization, M.T. and G.L.; methodology, M.T.; software, M.T. and S.Z.; validation, S.Z. and X.H.; formal analysis, M.T. and X.H.; investigation, M.T. and Y.L. (Yongjun Liu); resources, S.Z.; data curation, M.T.; writing—original draft preparation, M.T.; writing-review and editing, M.T., X.H. and Y.L. (Yunpeng Li); visualization, X.H.; supervision, Y.L. (Yongjun Liu); project administration, G.L.; funding acquisition, G.L. All authors have read and agreed to the published version of the manuscript.

Funding: This work was partially supported by the National Natural Science Foundation of China under Grant 61621005 and 62001352, the National Key R\&D Program of China under Grant 2016YFE0200400 and 2018YFB2202500, and the Key R\&D Program of Shaanxi Province under Grant 2017KW-ZD-12.

Data Availability Statement: Restrictions apply to the availability of these data. Data was obtained from China Academy of Space Technology (CAST) and are available from the authors with the permission of CAST.

Acknowledgments: The authors would like to thank the anonymous reviewers for their valuable and useful comments and suggestions that helped improve the paper. 
Conflicts of Interest: The authors declare no conflict of interest.

\section{References}

1. Zhu, S.; Liao, G.; Qu, Y.; Zhou, Z.; Liu, X. Ground moving targets imaging algorithm for synthetic aperture radar. IEEE Trans. Geosci. Remote Sens. 2011, 49, 462-477. [CrossRef]

2. Bovenga, F.; Derauw, D.; Rana, F.M.; Barbier, C.; Refice, A.; Veneziani, N.; Vitulli, R. Multi-chromatic analysis of SAR images for coherent target detection. Remote Sens. 2014, 6, 8822-8843. [CrossRef]

3. Filippo, B. COSMO-SkyMed staring spotlight SAR data for micro-motion and inclination angle estimation of ships by pixel tracking and convex optimization. Remote Sens. 2019, 11, 766. [CrossRef]

4. Wan, J.; Zhou, Y.; Zhang, L.; Chen, Z. A doppler ambiguity tolerated method for radar sensor maneuvering target focusing and detection. IEEE Sens. J. 2019, 19, 6691-6704. [CrossRef]

5. Huang, Y.; Liao, G.; Xu, J.; Li, J.; Yang, D. GMTI and parameter estimation for MIMO SAR system via fast interferometry RPCA method. IEEE Trans. Geosci. Remote Sens. 2018, 56, 1774-1787. [CrossRef]

6. Zhang, X.; Liao, G.; Zhu, S.; Zeng, C.; Shu, Y. Geometry-information-aided efficient radial velocity estimation for moving target imaging and location based on Radon transform. IEEE Trans. Geosci. Remote Sens. 2015, 53, 1105-1117. [CrossRef]

7. Li, D.; Zhan, M.; Liu, H.; Liao, Y.; Liao, G. A Robust Translational Motion Compensation Method for ISAR Imaging Based on Keystone Transform and Fractional Fourier Transform Under Low SNR Environment. IEEE Trans. Aerosp. Electron. Syst. 2017, 53, 2140-2156. [CrossRef]

8. Tian, M.; Liao, G.; Zhu, S.; He, X.; Li, Y. A novel method for high-speed maneuvering target detection and motion parameters estimation. Multidimens. Syst. Signal Process. 2020, 4, 1625-1647. [CrossRef]

9. Li, D.; Ma, H.; Liu, H.; Chen, Z.; Yang, Z. An efficient ground manoeuvring target refocusing method based on principal component analysis and motion parameter estimation. Remote Sens. 2020, 12, 378. [CrossRef]

10. Perry, R.P.; DiPietro, R.C.; Fante, R.L. SAR imaging of moving targets. IEEE Trans. Aerosp. Electron. Syst. 1999, 35, 188-200. [CrossRef]

11. Zhu, D.; Li, Y.; Zhu, Z. A keystone transform without interpolation for SAR ground moving-target imaging. IEEE Geosci. Remote Sens. Lett. 2007, 4, 18-22. [CrossRef]

12. Li, G.; Xia, X.; Peng, Y. Doppler keystone transform: An approach suitable for parallel implementation of SAR moving target imaging. IEEE Geosci. Remote Sens. Lett. 2008, 5, 573-577. [CrossRef]

13. Sun, Z.; Li, X.; Yi, W.; Gui, G. Detection of weak maneuvering target based on keystone transform and matched filtering process Signal Process. 2017, 140, 127-138. [CrossRef]

14. Huang, P.; Liao, G.; Yang, Z.; Xia, X.; Ma, J.; Zhang, X. An approach for refocusing of ground moving target without target motion parameter estimation. IEEE Trans. Geosci. Remote Sens. 2017, 55, 336-350. [CrossRef]

15. Rao, X.; Tao, H.; Su, J.; Guo, X.; Zhang, J. Axis rotation MTD algorithm for weak target detection. Digit. Signal Process. 2014, 26, 81-86. [CrossRef]

16. Xu, J.; Yu, J.; Peng, Y.; Xia, X. Radon-Fourier transform for radar target detection, I: Generalized Doppler filter bank. IEEE Trans. Aerosp. Electron. Syst. 2011, 47, 1186-1202. [CrossRef]

17. Xu, J.; Yu, J.; Peng, Y.; Xia, X. Radon-Fourier transform for radar target detection (II): Blind speed sidelobe suppression. IEEE Trans. Aerosp. Electron. Syst. 2011, 47, 2473-2489. [CrossRef]

18. Yu, J.; Xu, J.; Peng, Y.; Xia, X. Radon-Fourier transform for radar target detection (III): Optimality and fast implementations. IEEE Trans. Aerosp. Electron. Syst. 2012, 48, 991-1004. [CrossRef]

19. Zheng, J.; Su, T.; Liu, H.; Liao, G. Radar high-speed target detection based on the frequency-domain deramp-keystone transform IEEE J. Sel. Top. Appl. Earth Obs. Remote Sens. 2016, 9, 285-294. [CrossRef]

20. Zhou, F.; Wu, R.; Xing, M.; Bao, Z. Approach for single channel SAR ground moving target imaging and motion parameter estimation. IET Radar Sonar Navig. 2007, 1, 59-66. [CrossRef]

21. Kirkland, D. Imaging moving targets using the second-order keystone transform. IET Radar Sonar Navig. 2011, 5, 902-910. [CrossRef]

22. Tian, J.; Cui, W.; Xia, X.; Wu, S. A novel method for parameter estimation of space moving targets. IEEE Geosci. Remote Sens. Lett. 2013, 11, 389-393. [CrossRef]

23. Chen, X.; Guan, J.; Liu, N.; He, Y. Maneuvering target detection via Radon-fractional Fourier transform-based long-time coherent integration. IEEE Trans. Signal Process. 2014, 62, 939-953. [CrossRef]

24. Huang, P.; Liao, G.; Yang, Z.; Xia, X.; Ma, J.; Zhang, X. A fast SAR imaging method for ground moving target using a second-order WVD transform. IEEE Trans. Geosci. Remote Sens. 2016, 54, 1940-1956. [CrossRef]

25. Tian, M.; Liao, G.; Zhu, S.; Liu, Y.; He, X.; Li, Y. Long-time coherent integration and motion parameters estimation of radar moving target with unknown entry/departure time based on SAF-WLVT. Digit. Signal Process. 2020, 107, 102854. [CrossRef]

26. Li, X.; Sun, Z.; Yi, W.; Cui, G.; Kong, L.; Yang, X. Computationally efficient coherent detection and parameter estimation algorithm for maneuvering target. Signal Process. 2019, 155, 130-142. [CrossRef]

27. He, X.; Liao, G.; Zhu, S.; Xu, J.; Guo, Y.; Wei, J. Fast non-searching method for ground moving target refocusing and motion parameters estimation. Digit. Signal Process. 2018, 79, 152-163. [CrossRef] 
28. Xu, J.; Xia, X.; Peng, S.; Yu, J.; Peng, Y.; Qian, L. Radar maneuvering target motion estimation based on generalized Radon-Fourier transform. IEEE Trans. Signal Process. 2012, 60, 6190-6201.

29. Huang, P.; Liao, G.; Yang, Z.; Xia, X.; Ma, J. Long-time coherent integration for weak maneuvering target detection and high-order motion parameter estimation based on keystone transform. IEEE Trans. Signal Process. 2015, 64, 4013-4026. [CrossRef]

30. Kong, L.; Li, X.; Cui, G.; Yi, W.; Yang, Y. Coherent integration algorithm for a maneuvering target with high-order range migration. IEEE Trans. Signal Process. 2015, 63, 4474-4486. [CrossRef]

31. Huang, P.; Liao, G.; Yang, Z.; Xia, X.; Ma, J.; Zheng, J. Ground maneuvering target imaging and high-order motion parameter estimation based on second-order keystone and generalized Hough-HAF transform. IEEE Trans. Geosci. Remote Sens. 2017, 55, 320-335. [CrossRef]

32. Huang, P.; Xia, X.; Liao, G.; Yang, Z.; Zhang, Y. Long-Time Coherent Integration Algorithm for Radar Maneuvering Weak Target with Acceleration Rate. IEEE Trans. Geosci. Remote Sens. 2019, 57, 3528-3542. [CrossRef]

33. Li, X.; Cui, G.; Yi, W.; Kong, L. Fast coherent integration for maneuvering target with high-order range migration via TRT-SKT-LVD. IEEE Trans. Aerosp. Electron. Syst. 2016, 52, 2803-2814. [CrossRef]

34. Li, X.; Cui, G.; Kong, L.; Yi, W. Fast non-searching method for maneuvering target detection and motion parameters estimation. IEEE Trans. Signal Process. 2016, 64, 2232-2244. [CrossRef]

35. Fang, X.; Min, R.; Cao, Z.; Pi, Y. High-order RM and DFM correction method for long-time coherent integration of highly maneuvering target. Signal Process. 2019, 162, 221-233. [CrossRef]

36. Sun, G.; Xing, M.; Xia, X.; Wu, Y.; Bao, Z. Robust ground moving-target imaging using deramp-keystone processing. IEEE Trans. Geosci. Remote Sens. 2013, 51, 966-982. [CrossRef]

37. Chen, Z.; Zhou, Y.; Zhang, L.; Lin, C.; Huang, Y.; Tang, S. Ground moving target imaging and analysis for near-space hypersonic vehicle-borne synthetic aperture radar system with squint angle. Remote Sens. 2018, 10, 1996. [CrossRef]

38. Xin, Z.; Liao, G.; Yang, Z.; Huang, P.; Ma, J. A fast ground moving target focusing method based on first-order discrete polynomial-phase transform. Digit. Signal Process. 2017, 60, 287-295. [CrossRef]

39. Yu, W.; Su, W.; Gu, H. Ground maneuvering target detection based on discrete polynomial-phase transform and Lv's distribution. Signal Process. 2018, 144, 364-372. [CrossRef]

40. Su, J.; Xing, M.; Wang, G.; Bao, Z. High-speed multi-target detection with narrowband radar. IET Radar Sonar Navig. 2010, 4, 595-603. [CrossRef]

41. DiPietro, R.C. Extended factored space-time processing for airborne radar systems. In Proceedings of the 26th Asilomar Conference on Signals, Systems \& Computers, Pacific Grove, CA, USA, 26-28 October 1992; pp. 425-430. 